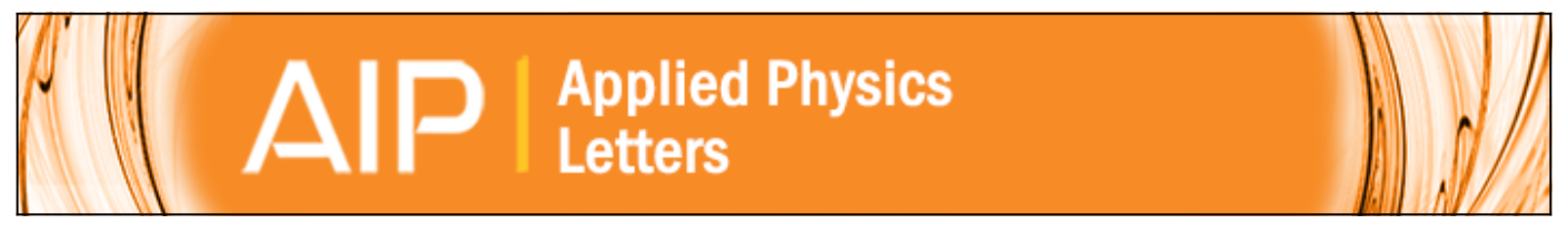

Temperature dependence of terahertz optical transitions from boron and phosphorus dopant impurities in silicon

S. A. Lynch, P. Townsend, G. Matmon, D. J. Paul, M. Bain, H. S. Gamble, J. Zhang, Z. Ikonic, R. W. Kelsall, and P. Harrison

Citation: Applied Physics Letters 87, 101114 (2005); doi: 10.1063/1.2042544

View online: http://dx.doi.org/10.1063/1.2042544

View Table of Contents: http://scitation.aip.org/content/aip/journal/apl/87/10?ver=pdfcov

Published by the AIP Publishing 


\title{
Temperature dependence of terahertz optical transitions from boron and phosphorus dopant impurities in silicon
}

\author{
S. A. Lynch, ${ }^{\text {a) }}$ P. Townsend, G. Matmon, and D. J. Paul' ${ }^{\text {b) }}$ \\ University of Cambridge, Cavendish Laboratory, Madingley Road, Cambridge, CB3 OHE, United Kingdom \\ M. Bain and H. S. Gamble \\ Queens University, School of Electrical and Electronic Engineering, Ashby Building, Stranmillis Road, \\ Belfast BT9 5AH, United Kingdom \\ J. Zhang \\ Imperial College, EXSS, Blackett Laboratory, Imperial College London, Prince Consort Road, \\ London SW7 2BW, United Kingdom \\ Z. Ikonic, R. W. Kelsall, and P. Harrison \\ University of Leeds, Institute for Microwaves and Photonics, School of Electronic and Electrical \\ Engineering, Leeds, LS2 9JT, United Kingdom
}

(Received 3 May 2005; accepted 26 June 2005; published online 2 September 2005)

\begin{abstract}
There has been considerable interest in using impurity dopants in silicon, such as boron and phosphorus, since it was demonstrated that such materials can show emission in the THz region of the electromagnetic spectrum. The measured temperature dependence of these transitions in this letter shows that the most optimistic maximum operating temperature of such an impurity-based laser is $90 \mathrm{~K}$. In a real device with current flowing and associated Joule heating, the actual operating range is likely to be much narrower and it is therefore unlikely that such a device would have a
\end{abstract} maximum operating temperature above 40 K. (c) 2005 American Institute of Physics.

[DOI: $10.1063 / 1.2042544]$

Until recently, the majority of semiconductor lasers have been realized in the III-V material system. There have, however, been sustained efforts over the past few years to develop a silicon-based laser source. Such a device would represent a considerable advancement in optoelectronic technology since it would open the way for inexpensive monolithic integrated optical and electronic components. In the last two years, there has been considerable interest in impurity dopants, such as boron and phosphorus, since it was demonstrated that such materials can show emission at $\mathrm{THz}$ frequencies. ${ }^{1-5}$ We investigated the temperature dependence of these impurity transitions in order to estimate the most optimistic operating range of an impurity-based silicon laser. These results should also be taken into account when designing potential $\mathrm{THz}$ silicon waveguides since both boron and phosphorus are commonly used to fabricate $n$ - and $p$-type Ohmic contacts and could contribute to potential optical losses.

Figure 1 shows two THz electroluminescence spectra for [Fig. 1(a)] a sample containing boron and [Fig. 1(b)] a sample containing phosphorus. In both cases, the samples in question were designed with a different purpose in mind. They were both grown with the intention of demonstrating $\mathrm{THz}$ intersubband emission from a $\mathrm{Si} / \mathrm{SiGe}$ quantum cascade active region. Under certain bias conditions however, several sharp features were observed in the electroluminescence spectra which were attributed to impurity transitions. The samples in Fig. 1 consisted of $4 \mu \mathrm{m}$ of a Si/SiGe superlattice sandwiched between $200 \mathrm{~nm} p$-doped $\left(5 \times 10^{18} \mathrm{~cm}^{-3}\right)$ contact layers, grown on top of $4 \mu \mathrm{m}$ of a SiGe virtual sub-

\footnotetext{
a) Electronic mail: sal44@cam.ac.uk

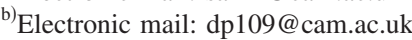

strate, which in turn was grown on top of a standard lightly $p$-doped silicon substrate $\left(2 \times 10^{15} \mathrm{~cm}^{-3}\right)$. The main difference between Figs. 1(b) and Fig. 1(a) was that the superlattice active region was grown on top of a buried tungsten silicide layer. A detailed description of how this buried silicide layer was realized can be found in Ref. 6. In this particular case, the handle wafers used in the fabrication of the buried silicide layer were lightly $n$ doped with phosphorus at a carrier concentration of $1 \times 10^{15} \mathrm{~cm}^{-3}$. Figure 1 shows several strong sharp spectral features in the electroluminescence spectrum which can be identified by comparison with absorption spectra in Ref. 7. In the case of the phosphorus-

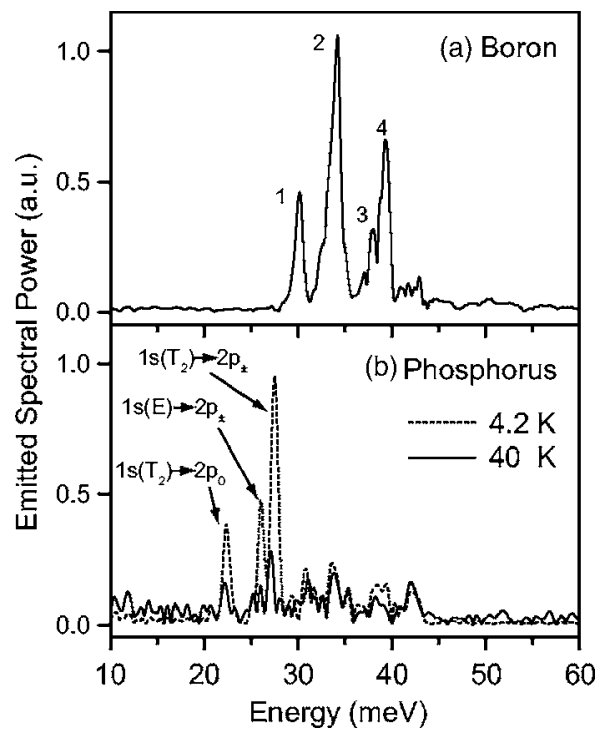

FIG. 1. THz electroluminescence spectra for (a) a sample containing boron and (b) a sample containing phosphorus. 
TABLE I. Wafer labels and corresponding resistivity and doping concentrations ( $\mathrm{P}$ - phosphorus and $\mathrm{B}$-boron).

\begin{tabular}{ccc}
\hline \hline Wafer label & Resistivity $(\Omega \mathrm{cm})$ & Doping concentration $\left(\mathrm{cm}^{-3}\right)$ \\
\hline P1 & $0.005-0.018$ & $1 \times 10^{19}$ \\
P2 & $0.04-0.14$ & $2 \times 10^{17}$ \\
P3 & 0.3 & $1.5 \times 10^{16}$ \\
P4 & $2-9$ & $5 \times 10^{15}$ \\
B1 & 0.02 & $5 \times 10^{18}$ \\
B2 & 0.05 & $1 \times 10^{18}$ \\
B3 & $10-20$ & $1 \times 10^{15}$ \\
\hline \hline
\end{tabular}

doped sample, impurity-based electroluminescence was observed up to about $40 \mathrm{~K}$.

In order to further assess the effect of temperature and dopant concentration on these transitions, we performed a series of absorption measurements on bulk-doped silicon wafers. Seven wafers were selected with different concentrations of boron and phosphorus. The choice of wafers was determined by what was available in our stocks. The details of each wafer are summarized in Table I. Phosphorus-doped wafers are labeled P1-P4, and boron-doped wafers are labeled B1-B3. A $1 \mathrm{~cm}^{2}$ sample was cut from each wafer and the bottom flat was polished at an angle of $1^{\circ}$ to the top flat. This was to avoid Fabry-Perot reflections from parallel flat surfaces. Each sample was stuck down with high thermal conductivity silver dag to a copper heatsink with an $8 \mathrm{~mm}$ hole for optical transmission. The heatsink was attached to the cold finger of a continuous flow cryostat allowing temperature tuning in the range of $4.2 \mathrm{~K} \leqslant T \leqslant 300 \mathrm{~K}$. Measurements were made using a Bruker 66VS spectrometer and a QMC helium cooled composite Si-bolometer detector.

The total absorption of each wafer was first measured as a function of temperature. No transmitted signal was recorded through samples with dopant concentrations greater than $10^{18} \mathrm{~cm}^{-3}$. The next highest dopant concentration was wafer P2 with a phosphorus concentration at $2 \times 10^{17} \mathrm{~cm}^{-3}$. The transmitted signal through this wafer was less than $1 \%$. Very little evidence of any distinct spectral features was observed. This is not surprising since at dopant concentrations greater than $10^{17} \mathrm{~cm}^{-3}$ and at cryogenic temperatures, the wave functions describing the energy states become sufficiently delocalized that the behavior of the material becomes more metallic and sharp spectral features become smeared out. The behavior of the samples with dopant concentrations in the range of $10^{15}-10^{16} \mathrm{~cm}^{-3}$ is markedly different. Figure 2 shows the total absorption as a function of temperature of Sample P3 (phosphorus) and Sample B3 (boron), in the temperature range of $4.2 \mathrm{~K} \leqslant T \leqslant 200 \mathrm{~K}$. The total absorption for boron does not show a large change over the temperature range in question. The maximum absorption is at $4.2 \mathrm{~K}$ and the absorption decreases with increasing temperature up to about $100 \mathrm{~K}$. Above this temperature the absorption begins to increase again as more carriers become available to undergo free-carrier absorption. This behavior can be easily explained with reference to Fig. 3. At very low heat sink temperatures, three very sharp absorption features can be observed between 30-40 meV. These correspond to Lines 1, 2, and 4 of the well-known $p_{3 / 2}$ series of $\operatorname{Si}(B){ }^{7,8}$ As the heatsink temperature is raised between $4.2 \mathrm{~K}$ and $90 \mathrm{~K}$, the transitions become progressively weaker, until they are finally extinguished around $100 \mathrm{~K}$. This corresponds closely with a

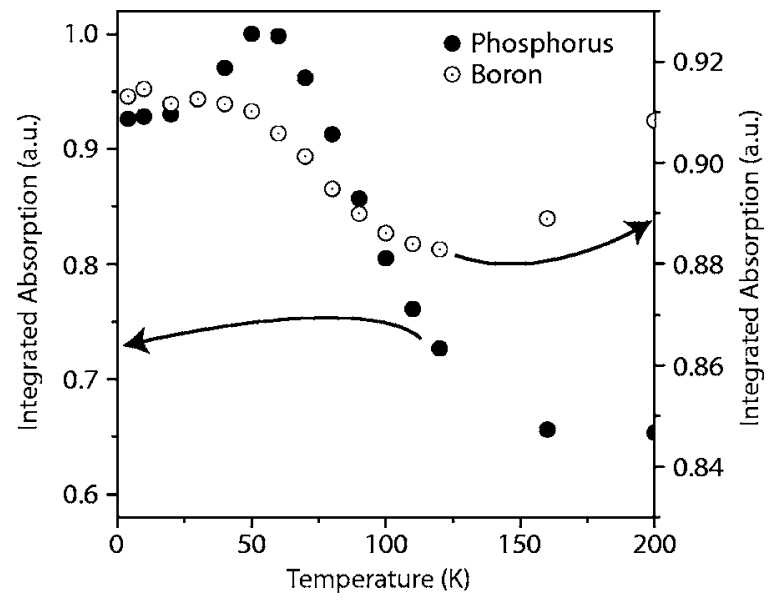

FIG. 2. Integrated absorption for samples P3 (phosphorus at 1.5 $\times 10^{16} \mathrm{~cm}^{-3}$, and B3 (boron at $1 \times 10^{15} \mathrm{~cm}^{-3}$ ), in the spectral range of $100-500 \mathrm{~cm}^{-1}$

reduction in the total absorption up to $100 \mathrm{~K}$ as shown in Fig. 2. In contrast, the behavior of the phosphorus-doped sample in Figure 2 is quite different. Unlike the boron-doped sample, the maximum absorption of the phosphorus doped sample is not at $4.2 \mathrm{~K}$, and there is a local maximum in the absorption with the temperature curve at $50 \mathrm{~K}$. A close examination of Fig. 4 reveals that the absorption spectrum is dominated by three features in the range of $30-45 \mathrm{meV}$ at $4.2 \mathrm{~K}$. These correspond to the $2 p_{0}, 2 p_{ \pm}$, and $3 p_{ \pm}$Lyman series transitions to the ground state $1 s\left(A_{1}\right){ }^{7}$ As the heatsink temperature is increased, a further set of thermally activated absorption lines appear between 20 and $30 \mathrm{meV}$. By considering the binding energies in Ref. 7 , it is possible to show that these transitions are between the $2 p_{0}, 2 p_{ \pm}$energy levels

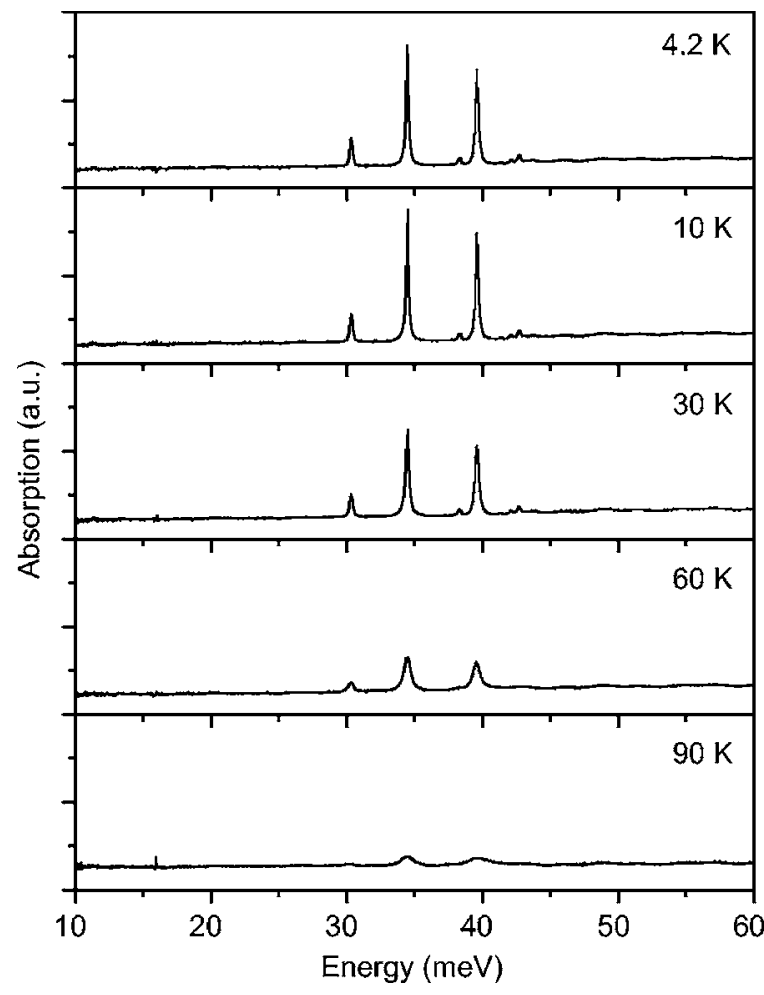

FIG. 3. THz absorption from boron doped silicon wafer B3 with temperature. The wafer has a resistivity of $10-20 \Omega \mathrm{cm}$, giving a boron concentration of $1 \times 10^{15} \mathrm{~cm}^{-3}$. 


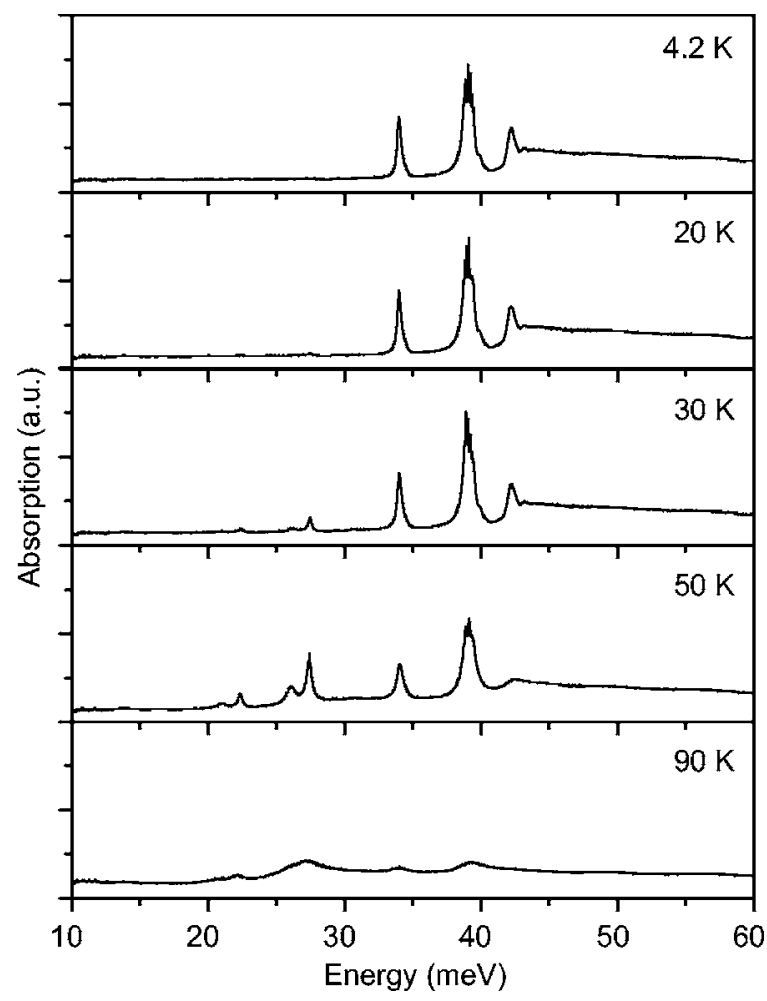

FIG. 4. THz absorption from phosphorus-doped silicon wafer P3 with temperature. The wafer has a resistivity of $0.3 \Omega \mathrm{cm}$, giving a phosphorus concentration of $1.5 \times 10^{16} \mathrm{~cm}^{-3}$.

and the excited ground state $1 s\left(T_{1}\right)$. These features reach maximum transition strength at $50 \mathrm{~K}$, the local maximum of the relevant absorption with the temperature curve in Fig. 2. It is from these additional thermally activated transitions that emission was observed in Fig. 1. Above $50 \mathrm{~K}$, the strength of all the transitions begins to weaken until they are extinguished at around $100 \mathrm{~K}$.

In conclusion, the temperature dependence of $\mathrm{THz}$ optical transitions from common dopants, boron and phosphorus, has been investigated. Sharp spectral features at $\mathrm{THz}$ frequencies resulting from these elements in single-crystal silicon were best observed in samples with bulk dopant concentrations in the range of $10^{15}-10^{16} \mathrm{~cm}^{-3}$. Above these dopant concentrations, the spectral features become very much broadened as the wave functions become delocalized. Below $10^{15} \mathrm{~cm}^{-3}$, the absorption becomes rapidly weaker and the spectral features become difficult to measure. The overall absorption due to boron in silicon decreases monotonically with increasing temperature in the temperature range of $4.2 \mathrm{~K} \leqslant T \leqslant 100 \mathrm{~K}$, as the transition strength of the boron lines becomes weaker and the transitions are eventually extinguished. The overall absorption due to phosphorus in silicon exhibits a local maximum at $50 \mathrm{~K}$ and then decreases with increasing temperature up to $100 \mathrm{~K}$. This local maximum corresponds with the observation of thermally activated transition lines which are not observed at lower temperatures. In each case, the exact transitions have been identified. These results are important and need to be taken into account when designing potential $\mathrm{THz}$ silicon waveguides since both boron and phosphorus are commonly used to realize $n$-and $p$-type Ohmic contacts and will contribute to potential losses. The measured temperature dependence of these transitions also shows that the most optimistic operating range of an impurity based laser is $4.2 \mathrm{~K} \leqslant T \leqslant 90 \mathrm{~K}$. This represents an ideal passive device with no current flowing. In a real device with current flowing and associated Joule heating, the actual operating range is likely to be much narrower and it is unlikely that such a device would have a maximum operating temperature above $40 \mathrm{~K}$ as in Fig. 1. This is quite consistent with the value quoted in Ref. 9.

This research was supported by U.S. DARPA under Air Force Contract No. F-19628-99-C-0074 and EU research Grant No. SHINE IST-2001-38035.

${ }^{1}$ S. A. Lynch, S. S. Dhillon, R. Bates, D. J. Paul, D. D. Arnone, D. J. Robbins, Z. Ikonic, R. W. Kelsall, P. Harrison, D. J. Norris, A. G. Cullis, C. R. Pidgeon, P. Murzyn, and A. Loudon, Mater. Sci. Eng., B 89, 12 (2002).

${ }^{2}$ R. Bates, S. A. Lynch, D. J. Paul, Z. Ikonic, R. W. Kelsall, P. Harrison, S. L. Liew, D. J. Norris, A. G. Cullis, W. R. Tribe, and D. D. Arnone, Appl. Phys. Lett. 83, 4092 (2003).

${ }^{3}$ T. N. Adam, R. T. Troeger, S. K. Ray, P.-C. Lv, and J. Kolodzey, Appl. Phys. Lett. 83, 1713 (2003).

${ }^{4}$ P.-C. Lv, R. T. Troeger, T. N. Adam, S. Kim, J. Kolodzey, I. N. Yassievich, M. A. Odnoblyudov, and M. S. Kagan, Appl. Phys. Lett. 85, 22 (2004).

${ }^{5}$ P.-C. Lv, R. T. Troeger, S. Kim, S. K. Ray, K. W. Goossen, J. Kolodzey, I. N. Yassievich, M. A. Odnoblyudov, and M. S. Kagan, Appl. Phys. Lett. 85, 3660 (2004).

${ }^{6}$ M. Bain, H. A. W. El Mubarek, J. M. Bonar, Y. Yang, O. Buiu, H. Gamble, B. M. Armstrong, P. L. F. Hemment, S. Hall, and P. Ashburn, IEEE Trans. Electron Devices 52, 317 (2005).

${ }^{7}$ C. Jagannath, Z. W. Grabowski, and A. K. Ramdas, Phys. Rev. B 23, 2082 (1981).

${ }^{8}$ A. K. Ramdas and S. Rodriguez, Rep. Prog. Phys. 44, 1297 (1981).

${ }^{9}$ S. G. Pavlov, H. W. Hubers, M. H. Rummeli, R. Kh. Zhukavin, E. E. Orlova, V. N. Shastin, and H. Riemann, Appl. Phys. Lett. 80, 4717 (2002). 